Research Article

\title{
Early Postnatal Care Service Utilization and Its Determinants among Women Who Gave Birth in the Last 6 Months in Wonago District, South Ethiopia: A Community-Based Cross-Sectional Study
}

\author{
Yordanos Tefera, ${ }^{1}$ Samirawit Hailu, ${ }^{1}$ and Ruth Tilahun $\mathbb{D D}^{2}$ \\ ${ }^{1}$ Department of Public Health, College of Health \& Medical Science, Dilla University, Dilla, Ethiopia \\ ${ }^{2}$ Department of Midwifery, College of Health \& Medical Science, Dilla University, Dilla, Ethiopia \\ Correspondence should be addressed to Ruth Tilahun; rutilahun@gmail.com \\ Received 26 June 2020; Revised 23 September 2020; Accepted 13 March 2021; Published 14 April 2021 \\ Academic Editor: George Uchenna Eleje
}

Copyright (C) 2021 Yordanos Tefera et al. This is an open access article distributed under the Creative Commons Attribution License, which permits unrestricted use, distribution, and reproduction in any medium, provided the original work is properly cited.

\begin{abstract}
Background. Postnatal care is one of the key strategies to reduce maternal and newborn morbidity and mortality. Early postnatal visit is especially the most critical time for survival of mothers and newborns, particularly through early detection and management of postpartum complication. Despite the benefits, most mothers and newborns do not receive postnatal care services from health care providers during the critical first few days after delivery. Objective. The aim of this study was to assess utilization of early postnatal care service and associated factors among women who gave birth in the last six months in Wonago District, Gedeo Zone, Southern Ethiopia. Methods. A community-based cross-sectional study design was employed at Wonago District. A total of 612 mothers who gave birth in the last six months were selected by simple random sampling technique. Pretested structured questionnaire was used for data collection. Data were entered into EpiData version 3.1 and then exported into SPSS version 20 for analysis. Principal component analysis (PCA) and bivariate and multivariate logistic regression were used. Result. In this study, 13.7\% of mothers utilized early postnatal care. Educational status of mothers (AOR = 3.7:95 CI; 1.3-10.7), place of delivery (AOR: 1.8:95 CI; 1.03-3.2), ANC attendance (AOR = 3.4:95 CI; 1.1-10.09), development of complication after delivery (AOR: 7.8:95 CI; 3.7-16.2), and previous history of postnatal care utilization (AOR: 2.1:95 CI; 1.13-3.9) were found to be associated with early postnatal care service utilization. Conclusion and Recommendations. Educational status of mothers, ANC attendance, place of delivery, delivery complication while giving recent birth, and past history of postnatal care utilization were significant predictors for early postnatal care utilization. Considering this, empowering women with education and overall strengthening of health facility to improve maternal health service utilization are necessary measures to be done at different levels to enhance early postnatal care utilization during this critical time.
\end{abstract}

\section{Background}

Postnatal care (PNC) is the care given to the mother and her newborn baby immediately after birth of baby up to the first six weeks of life, and it is decisive to the general wellbeing of both the mother and the newborn [1]. Three phases of contact are recommended which include the immediate postnatal period within the first 24 hours after birth, early postnatal period from day two through 7 , and late postnatal period extending from day 8 through 42 days [2].

PNC visit is very critical time for the survival of both mother and child. The early postnatal period, especially the first week after delivery, is the time when effective postnatal care can bring a difference to the health and life chances of mothers and newborns [3]. Since early postnatal care is very essential service to protect the mother from birth-related 
complications, much attention should be given by health care providers [4].

A large proportion of maternal and neonatal deaths occur during the 48 hours after delivery. Every year in Africa, at least 125,000 women and 870,000 newborns die in the first week after birth. It has been estimated that, 10 to 27 percent of newborn deaths could be averted if routine postnatal and curative care in the postnatal period reached 90 percent of babies and their mothers, which means that high PNC coverage could save up to 310,000 newborn lives each year in sub-Saharan Africa [5].

Despite the fact that early postnatal care visits are one the most important maternal health care interventions for prevention of morbidity and mortality, globally, only $48 \%$ of mothers are following the postnatal follow-up within two days of childbirth [5]. In developed countries, nearly all women and their infants receive postpartum and postnatal care. In developing countries, the need for care and support after birth was, until recently, less well recognized [2].

Evidence indicates that, in developing countries, proportion of women who had at least one postnatal care utilization within 42 days of delivery was 36\% [6]. Every day, 800 women die from maternal causes. Almost all of these deaths (99\%) occur in developing countries. One of the major indicators of the difference between developed and underdeveloped countries is maternal mortality. But this could easily be avoided as the necessary medical interventions exist and are well known. The key obstacle is the lack of access to quality care by pregnant women before, during, and after childbirth [7].

Most maternal and infant deaths occur in the first month after birth, almost half of postnatal maternal deaths occur within the first 24 hours, and $66 \%$ occur during the first week. 2.8 million newborns died in their first month of life; 1 million of these newborns died on the first day [8].

Higher number of maternal and newborn deaths occurs in the first week, especially on the first day. Early visits play a significant role in the prevention and management of many life threatening maternal and neonatal complications. This period is also the key time to promote healthy behaviors affecting women, newborns, and children, but the period following birth in Africa is often marked by cultural practices. Low coverage of care in the postnatal period negatively influences other maternal, newborn, and child health (MNCH) programs [9].

Approximately one-third of women in sub-Saharan Africa give birth in facilities, and no more than 13 percent of women who gave birth at home received a postnatal care visit within two days of delivery [10].

The 2016 Ethiopia Demographic Health Survey (EDHS) report stated that $\mathrm{PNC}$ service utilization is very much lower in Ethiopia; only $17 \%$ of women had received PNC during the first 2 days after birth. The proportion of women who received postnatal check-ups in the 2 days after delivery was 16.9\% SNNPR [11]. However, there is limited information about current utilization of postnatal care service in the study area. Therefore, this study is vital to visualize level of early PNC utilization and serve as an important tool for any possible interventions.

\section{Methods}

2.1. Study Area and Period. The study was conducted in Wonago District, which is one of the 6 districts in Gedeo Zone, SNNPRS (Southern Nations, Nationalities and Peoples Regional State) and Ethiopia. The district is found $377 \mathrm{~km}$ south of the capital city Addis Ababa and $102 \mathrm{~km}$ from the region capital Hawassa. The district has 21 kebeles (17 rural and 4 urban) and hosts a total population of 156,480 within 30,442 households. The dominant ethnic group in the district is Gedeo. Six health centers, 20 health posts, and 2 private clinics provide the overall health care service in the districts. The total population of districts is 149,165 . This study was conducted from December 5, 2017, to January 18, 2018.

2.2. Study Design and Population. Community-based crosssectional study was conducted among randomly selected mothers who gave birth in last six months preceding the data collection. Mentally and/or physically incapable mothers and those not living in the last six months in Wonago District were excluded from the study.

2.3. Sample Size Determination and Sampling Technique. The minimum required sample size of this study was determined using single population proportion formula. Proportion of postnatal care service utilization [12], margin of error, confidence interval, design effect, and nonresponse rate were assumed to be $23.7 \%, 5 \%, 95 \%, 2 \%$, and $10 \%$, respectively:

$$
\begin{aligned}
& n=\frac{\left((Z \alpha)^{2} / 2\right) * P(1-P)}{d^{2}} \\
& n=\frac{(1.96)^{2} * 0.237(1-0.237)}{(0.05)^{2}} 277 * 2=556 .
\end{aligned}
$$

Then, adding 10\% nonresponse rates resulted in the total sample required for the study being 612 .

Stratified sampling technique was used in order to obtain a representative sample. The kebeles were stratified into urban (2 kebeles) and rural (19 kebeles) settings then 8 kebeles (two from urban kebeles and 6 from rural kebeles) were selected randomly from the total 21 kebeles in the district. In each selected kebele, sampling frame which comprises a list of mothers who gave birth in the last six months and their full address was prepared with the help of HEW from health post registrations book (family folders).

From this sampling frame, a total sample size of 612 which was distributed based on proportional size allocation or proportional to the number of mothers who gave birth in the last six months prior to study in each kebele was traced to participate in the study. Finally, the study units (mothers who gave birth in last six months) in each $\mathrm{HH}$ were selected by random sampling technique.

2.4. Data Collection Tool and Procedure. Data were collected using structured questionnaires adapted and modified after 
reviewing different literature as appropriate so as to address the study objectives [13-15].

The main contents of questionnaire were sociodemographic characteristics, obstetric factor, maternal knowledge on PNC and danger sign on postpartum period, and health service-related factors.

Early postnatal care service utilization was measured as mothers who received at least one PNC service starting immediately after time of delivery up to end of first week. The data were collected by trained data collectors who had diploma in health-related fields and who were fluent in Gedeo-Offa and Amharic.

The questionnaire was carefully designed and prepared in English language first and then translated into local languages (Gedeo-Offa) and Amharic. To check for consistency, the questionnaire was further translated from the local languages (Gedeo-Offa) and Amharic to English. Testretest reliability was measured to determine the reliability of the tool and to measure its internal consistency; Cronbach's alpha was used, and it becomes 0.7 .

2.5. Data Quality Control. To assure the data quality, oneday training was given for data collectors and supervision was made at the time of data collection in each kebele; also, data collection tools were translated from English to Amharic and Gedeo-Offa by experts and backtranslated to English to check the consistency. Moreover, a pretest was done in Harsu kebele by taking $5 \%$ of the total sample size before one week of the actual data collection, and data were checked for completeness and corrective measures were taken immediately.

2.6. Data Processing and Analysis. Data were cleaned and entered using EpiData version 3.1 and then exported to SPSS version 20 for analysis. Data were analyzed using SPSS version 20. Descriptive statistics were computed. Binary and multivariate logistic regression analyses were done to see the association between the dependent and independent variables. Binary logistic regression was used to identify variables that are a candidate for multivariate logistic regression analysis at $p$ value $<0.25$, and multivariate logistic regression analysis was used to determine the factors that are independently associated with early postnatal care utilization at $p$ value $<0.05$ with a $95 \%$ confidence level. Finally, variables with $p$ value $<0.05$ were considered statistically significant. Model fitness was assessed through the Hosmer and Lemeshow test $(p=0.756)$.

\subsection{Variables of the Study}

2.7.1. Dependent Variable. The dependent variable is early postnatal care utilization.

\subsubsection{Independent Variable.}

(i) Sociodemographic characteristics: age, education, marital status, residence, occupation, education and occupation of the partner, and wealth index. (ii) Obstetric factors: gravidity, parity, birth order, ANC utilization, number of ANC visits, PNC utilization, timing of PNC visit, place for PNC, frequency of PNC, delivery complication after recent birth, and previous history of PNC.

(iii) Maternal knowledge on PNC: knowledge availability and benefits of PNC service, knowledge on danger sign of postpartum period, and knowledge on maternal and neonatal care on postpartum period.

(iv) Health service-related factor: time to reach health facility, advice/information provided by health care provider on postpartum danger sign, appointment for PNC during discharge, place of delivery, mode of delivery, and visit by HEW at home.

2.8. Operational Definitions. Early postnatal care utilization refers to mothers or their newborn babies who had at least one early postnatal care check-up for the current delivery by health care provider and health extension workers within first week of delivery.

2.9. Ethical Considerations. Ethical clearance to conduct this study was obtained from Dilla University, College of Health Science and Medicine Institutional Review Board. Written consent from each subject was asked and secured after detailed explanation of the main purpose of the study. All the provided information was strictly confidential, and written consent was taken. The participants had the right to withdraw from study at any time they want. The name of the respondent was not appearing on the questionnaire.

\section{Results}

3.1. Sociodemographic Characteristics of Mothers. The study included a total of 612 mothers who gave birth six months prior to the study with a response rate of $100 \%$. The mean age of mothers was 26.2 years $(\mathrm{SD}=4.9) .277(45.3 \%)$ were within the age group of 20-26 years. Almost all of the respondents, 600 (98\%), were married. About 477 (77.9\%) were living in rural area. Regarding educational status, more than half of the mothers, 348 (56.9\%), did not attend formal education and $512(83.7 \%)$ of them were housewives (Table $1)$.

3.2. Obstetric Characteristics of Mothers. Above half, 402 (65.7\%), of study participants had gravidity of $2-4$ and most mothers had two or more babies (multiparas). From the total of respondents, $483(78.9 \%)$ of the mothers had antenatal care follow-up during recent pregnancy (Table 2).

3.3. Knowledge of Mothers about PNC Service Utilization. Out of the total respondents, 465 (75.9\%) of mothers had ever heard of availability and importance of PNC services after delivery. About $54.6 \%$ of them had good knowledge of PPP (Figure 1). 
TABLE 1: Sociodemographic characteristics of mothers who gave birth in the last six months at Wonago District, South Ethiopia, $2017(n=612)$.

\begin{tabular}{lcc}
\hline Variables & Frequency & Percent $(\%)$ \\
\hline Age of mother at interval & 21 & \\
$\leq 19$ & 277 & 3.4 \\
$20-26$ & 252 & 41.2 \\
$27-32$ & 62 & 10.1 \\
$\geq 33$ & & \\
Marital status of mothers & 600 & 98 \\
Married & 12 & 2 \\
Others & & \\
Place of residence & 508 & 83 \\
Rural & 104 & 16.9 \\
Urban & & \\
\hline Educational status of mother & 348 & 56.9 \\
No formal education & 217 & 35.5 \\
Primary & 47 & 7.6 \\
Secondary and above & & \\
Educational status of father & 151 & 25.2 \\
No formal education & 328 & 54.6 \\
Primary & 121 & 20.2 \\
Secondary and above & & \\
\hline Occupational status of mother & 512 & 83.7 \\
House wife & 5 & 0.8 \\
Farmer & 19 & 3.1 \\
Daily labourer & 14 & 2.3 \\
Government employee & 62 & 33.3 \\
Merchant & 20.5 \\
\hline Occupational status of father & & \\
Farmer & & \\
Daily labourer & & \\
Government employee & & \\
Merchant & & \\
\hline Wealth index & & \\
Low & & \\
Middle & & \\
High & & \\
\hline
\end{tabular}

3.4. Utilization of Early Postnatal Care. Of all respondents, about 184 (30.1\%, 95 \% CI, 26.4\%-33.7\%) mothers had used postnatal care services after recent delivery and the level of early postnatal care service utilization was only $84(13.7 \%$, 95\% CI, $10.9 \%-16.4 \%$ ) of the 612 participants. Among mothers who got postnatal care service, almost all of the respondents stated that they got postnatal care services one time, and most, $112(60.3 \%)$, of postnatal care services users had got services at health center (Table 3).

3.5. Reason for Not Attending Early Postnatal Care Service. Absence of illness immediately after delivery and lack of knowledge on the benefits of early postnatal care service utilization were the most raised reasons for not utilizing early postnatal care service (Figure 2).

3.6. Early Postnatal Care Service Utilization and Health Service-Related Factors. From the responses, most of the respondents travel more than 30 minutes to reach the health
TABLE 2: Obstetric characteristics of mothers at Wonago District, South Ethiopia, $2017(n=612)$.

\begin{tabular}{lcc}
\hline Variables & Frequency & Percent (\%) \\
\hline Number of pregnancies & & \\
One & 82 & 13.4 \\
Two-four & 128 & 65.7 \\
Five and above & & 20.9 \\
\hline Number of alive births & \\
One & 402 & 13.4 \\
Two-four & 127 & 65.7 \\
Five and above & & 20.8 \\
\hline Birth order of last pregnancy & 83 & \\
First & 111 & 13.6 \\
Second & 225 & 18.1 \\
Third & 193 & 36.8 \\
Four and above & & 31.5 \\
\hline ANC visit & 483 & 78.9 \\
Yes & 129 & 21.1 \\
No & & \\
\hline Number of ANC visits $(n=483)$ & 109 & 22.5 \\
$\leq 2$ & 374 & 77.5 \\
$\geq 3$ & & 83 \\
\hline Past history of PNC & 104 & \\
Yes & 508 & \\
No & & \\
\hline & &
\end{tabular}

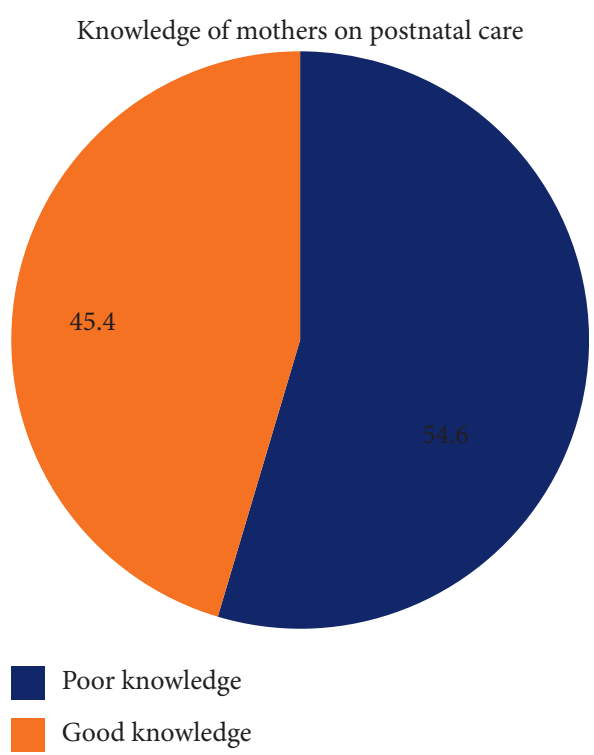

Figure 1: Knowledge about postnatal care utilization among mothers who gave birth in the last six months at Wonago District, South Ethiopia, 2017.

facility (252 (41.2\%)); $353(57.7 \%)$ gave birth in health center, from them only $129(49.8 \%)$ got an appointment for PNC, and only 137 (22.4\%) had got PNC service by health extension workers at their home (Table 4).

3.7. Factors Associated with Early Postnatal Care Service Utilization. All variables were assessed independently with the dependent variable in the bivariate logistic regression 
TABLE 3: Utilization of early postnatal care service among mothers who gave birth in the last six months at Wonago District, South Ethiopia, 2017.

\begin{tabular}{lcc}
\hline Variables & Frequency & Percent $(\%)$ \\
\hline PNC visit $(n=612)$ & 184 & 30.1 \\
Yes & 428 & 69.9 \\
No & 22 & 11.9 \\
\hline Time for PNC $(n=184)$ & 11 & 7.6 \\
Within 24 hours & 48 & 26 \\
$2-3$ days & 100 & 54.5 \\
$4-7$ days & & \\
$8-42$ days & 84 \\
Early PNC utilization $(n=84)$ & 528 \\
Yes & & 13.7 \\
No & 84 & 86.3 \\
\hline Frequency for PNC $(n=184)$ & 78 & 45.6 \\
One & 22 & 42.3 \\
Two & & 11.9 \\
Three and above & 41 & 22.3 \\
Place for PNC $(n=184)$ & 112 & 60.3 \\
Home & 31 & 16.9 \\
Health center & & \\
\hline
\end{tabular}

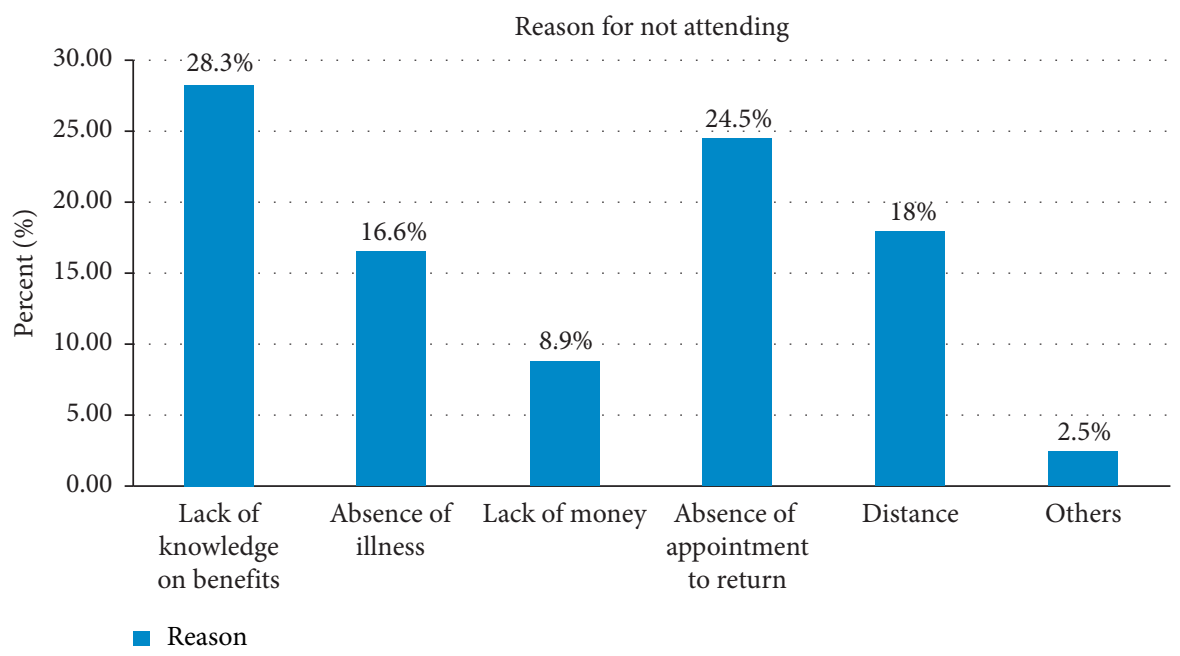

Figure 2: Respondents' reasons for not attending early postnatal care service among mothers who gave birth in the last six months at Wonago District, South Ethiopia, 2017.

analysis, and those variables having a $p$ value $<0.25$ were candidates for a multivariate logistic regression analysis (see Table 5). Then from multivariate logistic regression analysis at $p<0.055$, educational status of mothers (AOR: 3.7; 95\% CI: $1.3,10.6)$, place of delivery in the health institution (AOR 1.8, 95\% CI: 1, 3.2), experience of PNC utilization for previous child (AOR: 2.10; 95\% CI: 1.1, 3.9), ANC attendance before giving last birth, and having complication after giving recent birth were found to be statistically significant predictors for early postnatal care utilization.

The Hosmer-Lemeshow goodness of fit test $(p=0.085)$ provides evidence of model fitness with the predictors.
Those mothers who attended above secondary school were 3.7 times more likely to utilize the service than those who did not attend formal education (AOR: 3.7; 95\% CI: 1.3, 10.6).

Moreover, mothers who gave their last birth at health institution (AOR 1.8, 95\% CI: 1, 3.2) were two times more likely to got early postnatal care service utilization when compared to those mothers who gave birth at home.

Similarly, mothers who have at least one ANC attendance before giving their last birth were 3.4 times (AOR: 3.4, 95\% CI: 1.1, 10) more likely to utilize early service than those mothers who have no history of ANC attendance at all. 
Furthermore, mothers who develop complication after giving recent birth were 7.8 times more likely to get early postnatal care services utilization than mothers who did not develop complication after giving birth (AOR: 7.8, 95\% CI: 3.7, 16.2).

Likewise, previous experience of PNC has also been one of the associated factors with early PNC service utilization. Mothers with experience of postnatal care utilization for previous children were 2 times more likely to utilize early postnatal care than mothers who had no experience of postnatal care for previous child (AOR: 2.10 ; 95\% CI: 1.1, 3.9) (see Table 5).

\section{Discussion}

The study revealed that, among 612 postpartum mothers, the level of early PNC service utilization was 84 (13.7\%, 95\% CI, $10.9 \%-16.4 \%)$. This implies that substantial proportion of postpartum mothers did not use early PNC.

Proportion of mothers that used early PNC service in this study was much lower as compared with the finding in Nepal, where $43.2 \%$ of the mothers had attended immediate PNC [16]. This difference in service provision attributed to differences in socioeconomic status, geographical barriers, and accessibility of service, information, and education between countries.

Moreover, community-based cross-sectional study done in Debre Markos town in Ethiopia in 2015 revealed a higher figure which shows that $60.4 \%$ of mothers had got postnatal care services within 3-7 days following birth and in Arsis zone, $23.7 \%$ of mothers had attended early postnatal care service within one week of delivery $[12,17]$. This discrepancy might be because mothers of these study areas were less educated and less aware of the importance of postnatal care service utilization. In addition, it also might be due to difference in accessibility and availability of health service in urban area, since the majority of study participants lived in rural setting.

However, this study showed the early postnatal service utilization in the district to be slightly higher than the case of Mundri East Country, in South Sudan, where $11.4 \%$ of women used early PNC and 11.9\% in Tigray, 2014 [18, 19]. The attribute might be due to the study time period difference reported by Tigray and in this study there could be improvement in accessing and utilizing health care service through time and other factors attributed to place and social context variation between this study and previous studies. In addition, it might be also variation in study area and study subjects.

Mothers who attended secondary and above education were 3.7 times more likely to attend early PNC service utilization as compared with those who did not attend formal education. The finding is consistent with studies conducted in Nepal, Pakistan, Morocco, Kenya, Tanzania, South Sudan, and in Amhara region, Ethiopia [16, 19-22]. In addition, it is supported by local study conducted in Jebetina District, Amhara region, and study conducted in Abi-Adi Town, Tigray region $[18,23]$. This shows that education has a valuable input in order to empower women
TABLE 4: Health service-related factors of respondents among mothers who gave birth in that last six months at Wonago District, South Ethiopia, 2017.

\begin{tabular}{lcc}
\hline Variables & Frequency & Percent $(\%)$ \\
\hline Time to reach health facility $(n=612)$ & \\
$<15$ min & 194 & 31.7 \\
$15-30$ & 166 & 27.1 \\
$>30$ & 252 & 41.2 \\
\hline Place of delivery & \\
Home & 353 & 57.7 \\
Health center & 259 & 42.3 \\
\hline Get appointment for PNC $(N=259)$ & \\
Yes & 129 & 49.8 \\
No & 130 & 50.2 \\
\hline Advise on postpartum danger sign $(N=259)$ & \\
Yes & 116 & 44.8 \\
No & 143 & 55.2 \\
\hline Visit by HEW at home $(n=612)$ & 22.4 \\
Yes & 137 & 77.6 \\
No & 475 &
\end{tabular}

to gain access to health promotion message and information on how to obtain service and importance of available services. This is because educated mothers have an opportunity to participate in different social and economic positions and social empowerment.

Mothers who gave birth to their last child at health institution had $80 \%$ increased odds of getting early postnatal care service utilization when compared with mothers who gave birth to their last child at home. The finding was consistent with studies from Nepal, Pakistan, Kenya, Nigeria, Uganda, and Tanzania [20-22, 24, 25]. In addition to this, the result was nearly consistent with evidence from local finding in Jebitena District, Debre Markos Town, Lemo Woreda, Wolayita Zone, and Arsi Zone [12, 17, 23, 26, 27]. The explanation for this association was attributed to the fact that women who gave their last birth in health institution have greater opportunity to get exposed to health education and advice or counseling related to PNC service at the time of delivery. Besides this, they can get access to learn about the types, benefits, and availabilities of PNC services from skilled attendants during their stay in the health institutions.

Similarly, utilization of ANC has been found to positively influence early PNC service utilization. Mothers who had at least one attendance of ANC were more likely to utilize early PNC service than mothers who did not have ANC at all. This result was supported by the studies conducted in Gondar Zuria Districts, Amhara regions [28], Lemo Woreda, [27], Nepal [25], Benin, and Pakistan [22]. This is because mothers will have the opportunity to receive health education and counseling from health professionals about availability and benefit of PNC and schedules during their ANC follow-up. Attending ANC provides pregnant women the chance to obtain necessary health information on possible preparation for childbirth and advantage of delivering at health facility, and they generally get access to learn about the importance of utilization PNC service along with ANC examination. 
TABLE 5: Multivariable analysis of factors associated with early PNC utilization of mothers at Wonago District, South Ethiopia, 2017.

\begin{tabular}{|c|c|c|c|c|}
\hline \multirow{2}{*}{ Variables } & \multicolumn{2}{|c|}{ Early PNC attendance } & \multirow{2}{*}{$\begin{array}{c}\text { Crude } \\
\text { OR }(95 \% \mathrm{CI})\end{array}$} & \multirow{2}{*}{$\begin{array}{c}\text { Adjusted } \\
\text { OR }(95 \% \mathrm{CI})\end{array}$} \\
\hline & No $(\%)$ & Yes $(\%)$ & & \\
\hline \multicolumn{5}{|l|}{ Mothers education } \\
\hline No formal education & $317(91.1)$ & $31(8.9)$ & 1 & 1 \\
\hline Primary & $183(84.3)$ & $34(15.7)$ & $1.9(1.13-3.19)$ & $1.37(0.72-2.61)$ \\
\hline Secondary and above & $28(59.9)$ & $19(40.4)$ & $6.93(3.48-13.82)$ & $3.77(1.33-10.65) * *$ \\
\hline \multicolumn{5}{|l|}{ Husband education } \\
\hline No formal education & $136(92.5)$ & $11(7.5)$ & 1 & 1 \\
\hline Primary & $291(86.9)$ & $44(13.1)$ & $1.86(0.93-3.73)$ & $1.51(0.72-3.27)$ \\
\hline Secondary and above & $90(76.3)$ & $28(23.7)$ & $3.84(1.82-8.11)$ & $1.47(0.56-3.85)$ \\
\hline \multicolumn{5}{|l|}{ Occupation of mother } \\
\hline House wife & $451(88.1)$ & $61(11.9)$ & 1 & 1 \\
\hline Farmer & $21(87.5)$ & $3(12.5)$ & $1.05(0.30-3.64)$ & $0.89(0.23-3.44)$ \\
\hline Government employee & $9(64.3)$ & $5(35.7)$ & $4.10(1.33-12.65)$ & $1.64(0.41-6.43)$ \\
\hline Merchant & $47(75.8)$ & $15(24.2)$ & $2.36(1.24-4.47)$ & $1.26(0.58-2.77)$ \\
\hline \multicolumn{5}{|l|}{ Occupation of father } \\
\hline Farmer & $261(90.3)$ & $28(9.7)$ & 1 & 1 \\
\hline Government employee & $28(73.3)$ & $10(26.3)$ & $3.26(1.44-7.39)$ & $1.40(0.45-4.31)$ \\
\hline Merchant & $141(79.9)$ & $36(20.3)$ & $2.25(1.32-1.32)$ & $1.77(0.93-3.37)$ \\
\hline Daily labourer & $87(90.6)$ & $9(9.4)$ & $0.92(0.42-2.02)$ & $0.72(0.29-1.73)$ \\
\hline \multicolumn{5}{|l|}{ Having ANC } \\
\hline No & $125(96.9)$ & $4(3.1)$ & 1 & 1 \\
\hline Yes & $403(83.4)$ & $80(16.9)$ & $6.2(2.22-17.27)$ & $3.4(1.15-10.09) *$ \\
\hline \multicolumn{5}{|l|}{ Number of alive births } \\
\hline One & $67(80.7)$ & $16(19.3)$ & 1 & 1 \\
\hline Two-four & $346(86.6)$ & $56(13.9)$ & $0.67(0.36-1.25)$ & $0.75(0.21-2.74)$ \\
\hline Five & $115(90.6)$ & $12(9.4)$ & $0.43(0.19-0.97)$ & $1.01(0.34-2.96)$ \\
\hline \multicolumn{5}{|l|}{ Birth order } \\
\hline First & $67(80.75)$ & $16(19.3)$ & 1 & 1 \\
\hline Second & $94(84.7)$ & $17(15.3)$ & $0.75(0.35-1.60)$ & $1.82(0.39-8.46)$ \\
\hline Third & $190(84.4)$ & $35(15.6)$ & $0.77(0.40-1.48)$ & $2.45(0.55-10.82)$ \\
\hline Fourth and above & $177(91.7)$ & $16(8.3)$ & $0.37(0.17-0.80)$ & $1.06(0.36-3.12)$ \\
\hline \multicolumn{5}{|l|}{ Place of delivery } \\
\hline Home & $326(92.4)$ & $27(7.6)$ & 1 & 1 \\
\hline Health center & $202(78)$ & $57(22)$ & $3.4(2.08-5.56)$ & $1.8(1.03-3.26) *$ \\
\hline \multicolumn{5}{|l|}{ Delivery complication } \\
\hline No & $504(89.4)$ & $60(10.6)$ & 1 & 1 \\
\hline Yes & $24(50)$ & $24(50)$ & $8.4(4.49-15.7)$ & $7.83(3.76-16.28) * *$ \\
\hline \multicolumn{5}{|l|}{ Past history of PNC } \\
\hline No & $450(88.6)$ & $58(11.4)$ & 1 & 1 \\
\hline Yes & $78(75)$ & $26(25)$ & $2.58(1.53-4.35)$ & $2.10(1.33-3.90) * *$ \\
\hline \multicolumn{5}{|l|}{ HEW visit at home } \\
\hline No & $420(88.4)$ & $55(11.6)$ & 1 & 1 \\
\hline Yes & $108(78.8)$ & $29(21.2)$ & $2.05(1.24-3.37)$ & $0.77(0.42-1.39)$ \\
\hline
\end{tabular}

CI: confidence interval; OR: odds ratio; ANC: antenatal care; PNC: postnatal care; 1 : reference variable; ${ }^{*}$ significant at $p<0.05 ;{ }^{* *}$ significant at $p<0.01$.

Mothers who faced delivery complication after giving last birth were 7.8 times more likely to get early postnatal care services than mothers who did not face any complication after giving birth (AOR: 7.8, CI: 3.9-16.5). The finding agreed with the result of studies done in Debre Markos, Hadiya Zone, and Nepal in 2013 [17, 25, 27]. It is explained by the fact that exposure of complication increases fear of additional health complication; mothers who had experienced some danger sign were more likely to have increased perception of susceptibility and severity of the risks of maternal morbidity and mortality and which resulted in increased utilization of early postnatal service.

Mothers with experience of postnatal care for previous child were 2 times more likely to utilize early postnatal care than mothers who had no experience. This finding was consistent with cross-sectional study done in Gondar Zuria, in Addis Ababa town, and in Arsi zone [12, 15, 28]. This could be explained by the fact that experienced mothers had a better opportunity to get information/advice provision on the importance of early postnatal care service follow-up for 
both mother and newborn during previous childbirth from health care providers.

\section{Conclusion}

Less than one-fifth of the participants were using early postnatal care service. This figure was low as compared with most of the studies conducted in other parts of Ethiopia and other countries. Educational status of the mothers, place of delivery, delivery complication, and previous history of postnatal care were found to be significantly associated with early postnatal care service utilization.

\section{Data Availability}

The datasets used and/or analyzed during the current study are available from the corresponding author on reasonable request.

\section{Ethical Approval}

Ethical clearance was obtained from Dilla University College of Health and Medical Science Institutional Review Board.

\section{Consent}

Written consent was obtained from the study participants.

\section{Disclosure}

Confidentiality and privacy were maintained during data collection, analysis, and reporting.

\section{Conflicts of Interest}

The authors declare that they have no conflicts of interest.

\section{Authors' Contributions}

YT, SH, and RT have contributed to the design, data collection, data analysis, manuscript writing and development, editing, and revision of the final manuscript. All authors have read and approved the final manuscript.

\section{Acknowledgments}

The authors are very grateful to Wonago District Administration and Health Bureau for providing important information. Lastly, the authors would like to extend their deepest gratitude to the study participants.

\section{References}

[1] C. E. Warren, "Exploring the quality and effect of comprehensive postnatal care models in east and southern africa," 2015, http://www.iicrh.org.

[2] WHO, "Technical consultation on postpartum and postnatal Ca," WHO, 2010, http://www.who.int/making-pregnancysafer.

[3] WHO, WHO Recomendation On Postnatal Care Of The Mother And Newborn 2013, WHO, Geneva, Switzerland, 2013.
[4] WHO, Postpartum Care of the Mother and Newborn:a Practical Guidline, WHO, Geneva, Switzerland, 1998.

[5] N. Habiba, National Health Policy in Post Natal Care in Bangladesh: Gap between Policy and Practice, North South university, Dhaka, Bangladesh, 2012.

[6] USAID, Clinical Guidelines for Integrating Family Planning into Postpartum and Postabortion Care, USAID, Washington, DC, 2008 .

[7] WHO, World Health Stasistics2017: Monitoring Health for the SDGs, Sustainable Development Goal, WHO, Geneva, Switzerland, 2017.

[8] WHO, "Postnatal care for mothers and newborns highlights from the world health organization 2013 guidelines," WHO, Geneva, Switzerland, 2015.

[9] W. Charlotte and D. Pat, Opportunities for Africa'S Newborns Practical Data, Policy and Programmatic Supportfor New Born Care in Africa, Partenership For Matternal And Chiled Health, Geneva, Switzerland, 2006.

[10] B. Sines, U. Syed, S. Wall, and H. Worley, Postnatal Care: A Critical Opportunity to Save Mothers and Newborns, Save the childern, Geneva, Switzerland, 2007.

[11] Central Statistical Agency (CSA), Ethiopia Demographic and Health survey, CSA and ICF, Rockville, MD, USA, 2016.

[12] A. Teklehaymanot and D. Niguse, "Early postnatal care service utilization and associated factors among mothers who gave birth in the last 12 Months in aseko district, Arsi zone ,south East Ethiopia," Journal of Women's Health, vol. 9, 2017.

[13] WHO and UNFPA, Trends in Maternal Mortality: 1990 to 2015, WHO, Geneva, Switzerland, 2015.

[14] T. Mulatu, Assessment of Postnatal Care Utilization and Associated Factors in Asella Town, Addis Ababa, Ethopia, 2015.

[15] S. Berhanu, Assessment of Prevalence of Postnatal Care Utilization And Associated Factors Among Women Who Gave Birth And Attending Immunization Clinic In Selected Government Health Centers in Addis Ababa, Ethiopia, Addis Ababa University, Addis Ababa, Ethopia, 2015.

[16] M. Paudel, V. Khanal, B. Acharya, and M. Adhikarl, "Determinants of postnatal service utilization in a western district of Nepal," Women's Heal Care, vol. 18, 2013.

[17] M. Limenih, Z. Endale, and B. Assefa, "Postnatal care service utilization and associated factors among women in debre markos town , northwestern Ethiopia ," International Journal of Reproductive Medicine \& Gynecology, 2016.

[18] H. Alemayeh, H. Assefa, and Y. Adama, "Prevalence and Factors Associated With Post Natal Care Utilization In Abi Adi Town", Tigray, Ethiopia: a cross sectional study," International Journal of Pharmaceutical and Biological Sciences Fundamentals, vol. 8, no. 1, 2014.

[19] I. Jonathan and D. Grace, "Early postnatal care by postpartum mothers in Mundri East Country, South Sudan: izudi et al," BMC Health Services Research, vol. 17, 2017.

[20] J. Lwelamira and S. A. Safari J, "Utilization of maternal postnatal care services among women in selected villages of bahi district , Tanzania," Current Research in Social Sciences, vol. 7, 2016.

[21] D. Akunga and M. Kabue, "Determinants of postnatal care use in Kenya," African Popululation Study, vol. 28, 2014.

[22] A. Yunus, S. Iqbal, R. Munawar, and R. Zakar, "Determinants of postnatal care services utilization in Pakistan-insights from Pakistan demographic and health survey (PDHS) 2006-07," Middle-East Journal of Scientific Research, vol. 18, no. 10, pp. 1440-1447, 2013. 
[23] Y. Workineh and D. Hailu, "Factors affecting utilization of postnatal care service in Jabitena district, Amhara region, Ethiopia," Science Journal of Public Health, vol. 2, 2014.

[24] S. D. Oluwaseyi, Determinants of Postnatal Care Non-utilization Among Women in Nigeria, University of Witwatersrand, Johannesburg, South Africa, 2014.

[25] V. Khanal, M. Adhikari, R. Karkee, and T. Gavidia, "Factors associated with the utilisation of postnatal care services among the mothers of Nepal : BMC Women's Health," 2014, http://www.biomedcentral.com.

[26] W. Facha, M. Alemayehu, M. Meskele, and T. Fikadu, "Couples' opinion and women's utilization of postnatal care service in wolaita zone, Southern Ethiopia," Science Journal of Public Health, vol. 5, no. 4, 2017.

[27] T. Belachew, A. Taye, and T. Belachew and, "Postnatal care service utilization and associated factors among mothers," Journal of Women"s Health, vol. 5, no. 3, 2016.

[28] F. Tesfahun, W. Worku, F. Mazengya, and M. Kifle, "Knowledge, perception and utilization of postnatal care of mothers in gondar Zuria district, Ethiopia: a cross-sectional study," Maternal and Child Health Journal, vol. 18, 2014. 